\title{
Editorial: Recent Advances in Waste-Heat Harvesting via Thermoelectrics: From Theory to Materials and Devices
}

\author{
Muhammet S. Toprak ${ }^{1 *}$, Sedat Ballikaya ${ }^{2}$ and Emrah Celik ${ }^{3}$ \\ ${ }^{1}$ Department of Applied Physics, Royal Institute of Technology, Stockholm, Sweden, ${ }^{2}$ Department of Physics, Faculty of Science, \\ Istanbul University, Istanbul, Turkey, ${ }^{3}$ Department of Mechanical and Aerospace Engineering, University of Miami, Coral Gables, \\ FL, United States
}

Keywords: thermoelectric (TE), thermogenerator (TEG), nanostructure, severe plastic deformation (SPD), figure of merit (ZT)

\section{Editorial on the Research Topic}

Recent Advances in Waste-Heat Harvesting via Thermoelectrics: From Theory to Materials and Devices

Low-dimensional thermoelectric (TE) materials have been suggested to possess higher TE performance than their bulk counterparts through theoretical analysis and experimental explorations (Hicks and Dresselhaus, 1993; Dresselhaus et al., 1999). Therefore, intensive research has been devoted to the synthesis and processing optimization of TE materials to maintain the favorable micro-nanostructure in the resultant TE consolidates. Various materials architectures and top-down and bottom-up strategies have been investigated to identify the microstructure with the most favorable combination of transport properties. Based on the temperature range of operation, TE materials can be divided into three classes, namely ambient, mid-, and high-T materials, where the materials' bandgap and stability determine the viability of a particular composition as a TE material (Snyder and Toberer, 2010).

A serious problem in TE materials research has been great variations in the performance of the same material made through different routes. This difference can be an order of magnitude in the case of Bi-Te alloys, which is the most common TE material for applications at ambient temperature. The extent of variability in the TE performance has not been discussed in detail in the literature. Hamawandi et al. discusses this issue by comparing a solution chemical and a solid-state synthetic route and the resultant materials' microstructure and transport properties in detail for $\mathrm{Bi}_{2-\mathrm{x}} \mathrm{Sb}_{\mathrm{x}} \mathrm{Te}_{3}$ system. The study shows a textured nanostructure and better overall performance for the materials synthesized through the solution chemical route.

$\mathrm{Cu}_{2-\mathrm{x}} \mathrm{Se}$ has been considered a promising TE material due to its performance at intermediate temperature regions, however it has stability issues at elevated temperatures. Several strategies have been developed to enhance the $\mathrm{ZT}$ and the chemical stability of $\mathrm{Cu}_{2-\mathrm{X}} \mathrm{Se}$ over a wide temperature range. Various studies have proven that structural and TE transport properties of $\mathrm{Cu}_{2-\mathrm{x}} \mathrm{Se}$ can be modified with a variety of nano-inclusions and the synthesis route (Tafti et al., 2016; Ballikaya et al., 2019a; Ballikaya et al., 2019b; Hamawandi et al., 2020). Among these strategies, nanoscale incorporation of other elements and compounds was shown to be promising (Ballikaya et al., 2019b). Demirci et al. reports on the transport properties of nanostructured $\mathrm{Cu}_{2-\mathrm{x}} \mathrm{Se}$-skutterudite solid-solutions and discusses the results in terms of crystal mismatch of guest and host phases, concluding that skutterudite nanoparticle incorporation into $\mathrm{Cu}_{2-\mathrm{x}} \mathrm{Se}$ has a beneficial effect on TE performance. 
Si and SiGe are materials of interest for high-temperature TE energy conversion. Nanowires are 1-dimensional nanomaterials that have been used to increase the power factor and reduce the thermal conductivity of TE materials, resulting in significantly enhanced ZT values. $\mathrm{Li}$ et al. presents a review on $\mathrm{Si}$ and SiGe thermoelectric nanowires as proper candidates for microthermoelectric generators, discussing basic TE principles, materials, structures, fabrication, measurements, and applications.

When it comes to TE modules, leg geometry and mechanical stability of the materials are key issues for reliable and robust devices for TE applications. There is little knowledge about what geometries are beneficial in applications with differing thermal conditions. Şişik and LeBlanc investigates various leg shapes, by numerically modeling the thermal and electrical performance of bismuth telluride and silicon germanium alloys, to identify the best performing leg shape under constant temperature and heat flux boundary conditions. They underscore the importance of leg geometry on electrical and thermal performance of a TE leg, as

\section{REFERENCES}

Ballikaya, S., Oner, Y., Temel, T., Ozkal, B., Bailey, T. P., Toprak, M. S., et al. (2019a). Thermoelectric and thermal Stability Improvements in Nano-Cu2Se Included Ag2Se. J. Solid State. Chem. 273, 122-127. doi:10.1016/j.jssc.2019.02.037

Ballikaya, S., Sertkol, M., Oner, Y., Bailey, T. P., and Uher, C. (2019b). Fracture Structure and Thermoelectric Enhancement of Cu2Se with Substitution of Nanostructured Ag2Se. Phys. Chem. Chem. Phys. 21 (25), 13569-13577. doi:10.1039/c9cp00793h

Dresselhaus, M. S., Dresselhaus, G., Sun, X., Zhang, Z., Cronin, S. B., and Koga, T. (1999). Low-dimensional Thermoelectric Materials. Phys. Solid State. 41 (5), 679-682. doi:10.1134/1.1130849

Hamawandi, B., Ballikaya, S., Råsander, M., Halim, J., Vinciguerra, L., Rosén, J., et al. (2020). Composition Tuning of Nanostructured Binary Copper Selenides through Rapid Chemical Synthesis and Their Thermoelectric Property Evaluation. Nanomaterials 10, 854. doi:10.1021/acsaem.0c01525.s00110.3390/nano10050854

Hicks, L. D., and Dresselhaus, M. S. (1993). Effect of Quantum-Well Structures on the Thermoelectric Figure of merit. Phys. Rev. B. 47 (19), 12727-12731. doi:10.1103/physrevb.47.12727 well as the importance of considering the device operating condition when selecting the best leg shape.

TE leg materials with a high figure of merit, ZT, are the essential basis to build TE generators.

Severe plastic deformation via high-pressure torsion (HPT) was shown to result in very fine grains in micro- or even nano size with small and high angle grain boundaries and in parallel, which introduced a high level of defects. Rogl and Rogl presents a review, surveying the plastic deformation as a useful tool for mechanical strengthening of skutterudite and half-Heusler alloys.

We hope that the reader will find this research topic a useful reference in the field of thermoelectric materials and devices.

\section{AUTHOR CONTRIBUTIONS}

MT wrote the first draft. SB and EC provided critical comments and editorial suggestions for revisions. All the authors agreed on the submitted version.

Snyder, G. J., and Toberer, E. S. (2010). Complex Thermoelectric Materials. Mater. Sci. Eng. 7, 101-110. doi:10.1142/9789814317665_0016

Tafti, M. Y., Ballikaya, S., Khachatourian, A. M., Noroozi, M., Saleemi, M., Han, L., et al. (2016). Promising Bulk Nanostructured Cu2Se Thermoelectrics via High Throughput and Rapid Chemical Synthesis. RSC Adv. 6, 111457-111464. doi:10.1039/c6ra23005a

Conflict of Interest: The authors declare that the research was conducted in the absence of any commercial or financial relationships that could be construed as a potential conflict of interest.

Copyright (c) 2021 Toprak, Ballikaya and Celik. This is an open-access article distributed under the terms of the Creative Commons Attribution License (CC BY). The use, distribution or reproduction in other forums is permitted, provided the original author(s) and the copyright owner(s) are credited and that the original publication in this journal is cited, in accordance with accepted academic practice. No use, distribution or reproduction is permitted which does not comply with these terms. 